\title{
METABÓLITOS SECUNDÁRIOS DAS ESPONJAS Aplysina fistularis E Dysidea sp. E ATIVIDADE ANTITUBERCULOSE DA 11-CETOFISTULARINA-3
}

\author{
Renata C. Gandolfi, Marina B. Medina e Roberto G. S. Berlinck* \\ Instituto de Química de São Carlos, Universidade de São Paulo, CP 780, 13560-970 São Carlos - SP, Brasil \\ Simone P. Lira \\ Departamento de Ciências Exatas, Escola Superior de Agricultura “Luiz Queiroz”, Universidade de São Paulo, CP 9, 13418-900 \\ Piracicaba - SP, Brasil \\ Fabio Cícero de Sá Galetti e Célio L. Silva \\ Farmacore Biotecnologia Ltda, Ribeirão Preto - SP, / Faculdade de Medicina de Ribeirão Preto, Universidade de São Paulo, \\ Av. Bandeirantes, 3900, 14049-900 Ribeirão Preto - SP, Brasil \\ Katyuscya Veloso e Antonio G. Ferreira \\ Departamento de Química, Universidade Federal de São Carlos, CP 676, 13560-970 São Carlos - SP, Brasil \\ Eduardo Hajdu \\ Museu Nacional, Universidade Federal do Rio de Janeiro, Quinta da Boa Vista, s/n, 20940-040 Rio de Janeiro - RJ, Brasil \\ Solange Peixinho \\ Departamento de Biologia, Universidade Federal da Bahia, Campus Univ. Ondina, 40170-290 Salvador - BA, Brasil
}

Recebido em 1/12/09; aceito em 9/6/10; publicado na web em 24/9/10

\begin{abstract}
SECONDARY METABOLITES FROM THE SPONGES Aplysina fistularis AND Dysidea sp. AND THE ANTITUBERCULOSIS ACTIVITY OF 11-KETOFISTULARIN-3. The present investigation reports the isolation of aeroplysinin-2, 2-(3,5-dibromo4-methoxyphenyl)- $N, N, N$-trimethyletanamonium, 7,9-dibromo-10-hydroxy-8-methoxy-1-oxa-2-azaspiro[4.5]deca-2,6,8-trien3-carboxylic acid and its methyl ester, 11-oxoaerothionin, aerothionin, 11-keto-12-hydroxyaerothionin, 11-ketofistularin-3 and fistularin-3 from Aplysina fistularis, as well as of furodysinin lactone and $9 \alpha, 11 \alpha$-epoxicholest-7-en-3 $\beta, 5 \alpha, 6 \alpha, 10$-tetrol-6-acetate from Dysidea sp. Although the extracts of both sponges displayed antituberculosis activity, only 11-ketofistularin-3 isolated from $A$. fistularis displayed antimycobacterial activity against Mycobacterium tuberculosis $\mathrm{H} 34 \mathrm{Rv}$, with MIC at $16 \mu \mathrm{g} / \mathrm{mL}$ and SI of 40, a result that reinforce that fistularin-3 derivatives are interesting leads for the development of antituberculosis drugs.
\end{abstract}

Keywords: marine sponge; Aplysina fistularis; Dysidea sp..

\section{INTRODUÇÃO}

A tuberculose é uma doença respiratória prevalente em todo o mundo. Estima-se que um terço da população mundial esteja infectada com o agente etiológico causador da tuberculose, a micobactéria Mycobacterium tuberculosis. Considerada doença erradicada durante os anos 60-70, a tuberculose ressurgiu nos anos 80 associada a linhagens resistentes a antibióticos, de difícil tratamento, que exige 6 meses ininterruptos de quimioterapia. Considera-se que a tuberculose é um dos maiores problemas de saúde pública, especialmente quando associada com doenças que promovem deficiência de imunidade, tais como AIDS. ${ }^{1,2}$ Neste contexto, a busca por novos agentes terapêuticos para o tratamento da tuberculose tem chamado a atenção.

Dois dentre os cinco principais antibióticos utilizados no tratamento de tuberculose são de origem natural. A estreptomicina (1), originalmente isolada das actinobactérias Streptomyces lavendulae e $S$. griseus, atua sobre $M$. tuberculosis inibindo a síntese proteica por parte da subunidade ribossomal 30S. Já a rifampicina (2) é um derivado da rifamicina (3), a qual foi originalmente isolada de $S$. mediterranei. A rifampicina atua sobre $M$. tuberculosis inibindo a transcrição da subunidade $\beta$ da RNA polimerase. ${ }^{3}$ Além da estreptomicina e da rifampicina, dentre os sete antibióticos de segunda linha utilizados no tratamento de tuberculose, quatro são produtos naturais ou derivados de produtos naturais. ${ }^{3}$ Sendo assim, é evidente a importância de produtos do metabolismo se-

*e-mail: rgsberlinck@iqsc.usp.br cundário na descoberta e desenvolvimento de medicamentos para o tratamento da tuberculose. .-10 $^{4}$

A prospecção de extratos de invertebrados marinhos da costa do Brasil com atividade antituberculose ${ }^{11}$ levou-nos à descoberta de derivados da dibromotirosina, ${ }^{12}$ bem como de alcaloides alquilpiridínicos ${ }^{13}$ e alquilpiperidínico ${ }^{14}$ ativos contra $M$. tuberculosis H37Rv. A continuidade deste programa nos levou a investigar os extratos de duas esponjas marinhas, Aplysina fistularis e Dysidea sp., das quais os extratos metanólicos apresentaram atividade antituberculose contra M. tuberculosis H37Rv. O único composto ativo isolado, a 11-ceto-fistularina-3, apresentou potente atividade antituberculose e excelente índice de seletividade.

\section{RESULTADOS E DISCUSSÃO}

\section{Investigação química do extrato $\mathrm{MeOH}$ da esponja Aplysina fistularis}

O extrato metanólico de A. fistularis foi submetido a várias separações cromatográficas (ver Parte Experimental), resultando na obtenção dos derivados da dibromotirosina aeroplisinina-2 (4), ${ }^{15}$ 2-(3,5-dibromo-4-metoxifenil)- $N, N, N$-trimetiletanamônio (5), ${ }^{16}$ o ácido 7,9-dibromo-10-hidroxi-8-metoxi-1-oxa-2-azaspiro[4.5] deca-2,6,8-trieno-3-carboxílico (6), ${ }^{17}$ bem como o éster metílico deste último (7), ${ }^{18}$ a 11-oxoaerotionina (8), ${ }^{19}$ a aerotionina $(\mathbf{9}),{ }^{20} \mathrm{a}$ 11-ceto-12-hidroxiaerotionina (10), ${ }^{21}$ a 11-cetofistularina-3 (11) ${ }^{22} \mathrm{e}$ a fistularina-3 (12). ${ }^{23}$ Todos os derivados da dibromotirosina isolados de 
<smiles>C[C@@H]1O[C@@H](O[C@@H]2[C@@H](NC(=N)N)[C@H](O)[C@@H](NC(=N)N)[C@H](O)[C@H]2O)[C@H](O[C@H]2O[C@H](CO)[C@@H](O)[C@H](O)[C@H]2N(C)O)[C@]1(C)O</smiles>

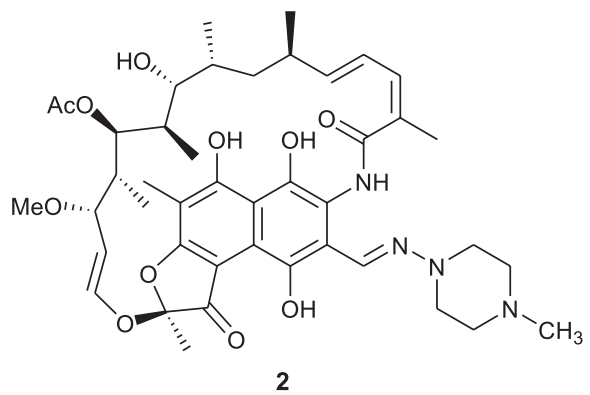

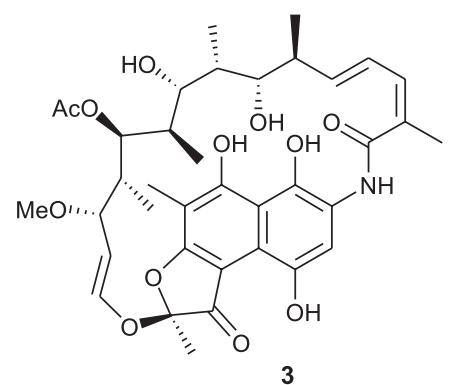

A. fistularis coletada na Baía de Todos os Santos foram identificados pela análise de seus dados espectroscópicos e por comparação com dados da literatura, cujas referências para comparação são indicadas para os compostos 4-12 na seção experimental.

Todos os compostos isolados de A. fistularis da Baía de Todos os Santos já foram isolados de outras esponjas marinhas da ordem Verongida, inclusive da costa do Brasil. A aeroplisinina-2 (4) foi por nós isolada anteriormente a partir de uma esponja do gênero Aplysina. ${ }^{24} \mathrm{O}$ 2-(3,5-dibromo-4-metoxifenil)- $N, N, N$-trimetiletanamônio (5) foi por nós anteriormente isolado da esponja Verongula gigantea.$^{25} \mathrm{O}$ ácido 6 e seu respectivo éster metílico 7 não foram anteriormente isolados de esponjas Verongida do Brasil, e sim das esponjas Verongula sp., ${ }^{16}$ Pseudoceratina sp. $^{17}$ e Verongula gigantea ${ }^{26}$ do Caribe. Todavia, o éster etílico de $\mathbf{6}$ foi por nós isolado de Aplysina sp. ${ }^{24}$ Embora o nome sistemático IUPAC 7,9-dibromo-10-hidroxi-8-metoxi-1-oxa2-azaspiro[4.5]deca-2,6,8-trieno-3-carboxílico possa ser utilizado para designar o composto 6, o grupo de Fattorusso não propôs outra designação para esta substância. ${ }^{16,17,26}$ Desta forma, propomos aqui o nome ácido verongidoico para 6 e éster metílico do ácido verongidoico para 7. Tal designação se justifica pelo fato de o ácido $\mathbf{6}$ ser a unidade estrutural mais comumente encontrada em derivados da dibromotirosina isolados de esponjas marinhas da ordem Verongida. A 11-oxoaerotionina (8) foi por nós anteriormente isolada da esponja A. fulva também da Baía de Todos os Santos, bem como a aerotionina (9) e a 11-oxo-12-hidroxiaerotionina (10). ${ }^{27}$ Já a 11-cetofistularina-3 (11) foi isolada anteriormente das esponjas Aplysina archeri $^{22}$ e Aplysina sp. ${ }^{28}$ Por fim, a fistularina-3 (12) foi por nós isolada a partir das esponjas A. cauliformis, ${ }^{12}$ A. fulva ${ }^{27,29}$ e A. caissara. ${ }^{30}$

Análises de dicroísmo circular realizadas com os compostos $\mathbf{4}$, 6-12 indicaram que as configurações absolutas dos centros estereogênicos C-1 e C-6, bem como C-1' e C-6' nos compostos 8-12 são todos
$1(R), 6(S)$ e 1'(R), 6' $(S)$. Estas análises foram realizadas e comparadas como anteriormente descrito para compostos correlatos isolados de $A$. caissara..$^{30}$ Os resultados obtidos das análises de dicroísmo circular reforçam, de maneira contundente, a proposta de rota biogenética por nós discutida para derivados da dibromotirosina que apresentam o ácido verongidoico (6) em sua estrutura. ${ }^{27}$

Estudos anteriores realizados com a esponja A. fistularis levaram ao isolamento da aerotionina $(\mathbf{9}),{ }^{31}$ bem como de uma série de derivados da dibromotirosina obtidos em estudos de biossíntese..$^{32,33}$ É importante ressaltar que espécies designadas Aplysina fistularis forma fulva ${ }^{24}$ e Aplysina fistularis-fulva ${ }^{34}$ se referem à espécie $A$. fulva, enquanto que Aplysina fistularis insularis ${ }^{35}$ se refere à espécie Aplysina insularis. Logo, as fistularinas-1, -2 e -3 foram originalmente isoladas de A. fulva (= A. fistularis forma fulva), mas não de A. fistularis ${ }^{23}$ Sendo assim, esta é a primeira investigação exaustiva dos metabólitos secundários da espécie $A$. fistularis.

Após o isolamento dos compostos 4-11 a partir de A. fistularis, estes foram testados contra M. tuberculosis H37Rv, mas somente a 11-cetofistularina-3 (11) apresentou atividade antimicobacteriana. A fistularina-3 (12) e a 11-desoxi-fistularina-3 (13) já haviam sido testadas contra M. tuberculosis H37Rv, e apresentaram MIC de 7,1 e $7,3 \mu \mathrm{g} / \mathrm{mL}$, respectivamente. Os índices de seletividade (IS $=\mathrm{IC}_{50} / \mathrm{MIC}$ ) dos compostos $\mathbf{1 2}$ e $\mathbf{1 3}$ contra macrófagos $\mathrm{J} 774$ foram de 28,17 e 86,13 , respectivamente. No caso da 11-cetofistularina-3 (11), esta apresentou MIC de $16 \mu \mathrm{g} / \mathrm{mL}$ e SI de 40, e esta é a primeira avaliação deste composto em bioensaio de atividade antituberculose. Estes dados reforçam nossa hipótese de que o estado de oxidação em C-11 na estrutura das fistularinas 11-13 pouco afeta a ação antimicobacteriana destes compostos. ${ }^{12}$ Considerando-se os excelentes índices de seletividade apresentados pelos compostos 11-13 frente a M. tuberculosis H37Rv, esta classe de substâncias constitui um bom modelo para o desenvolvimento de fármacos para o tratamento da tuberculose. Sendo assim, foi depositada patente no INPI para o possível desenvolvimento de fistularinas e derivados no tratamento de tuberculoses. ${ }^{36}$

\section{Investigação química da esponja Dysidea $\mathrm{sp.}$}

Esponjas do gênero Dysidea são, ao lado das esponjas da ordem Verongida, das mais estudadas do ponto de vista químico/farmacológico, ${ }^{37}$ e também do grupo de esponjas que vem sendo estudado há quase 40 anos. ${ }^{38}$ Metabólitos de classes diversas já foram isolados de esponjas marinhas do gênero Dysidea, os quais incluem fenóis bromados, terpenos, peptídeos modificados, derivados de ácidos graxos incomuns, neurotoxinas e alcaloides ${ }^{39}$ Recentemente reportamos os resultados das primeiras investigações químicas de esponjas do gênero Dysidea oriundas do litoral do Brasil, que resultaram no isolamento de ceramidas saturadas ${ }^{39} \mathrm{e}$ de derivados do ácido pirodisinoico. ${ }^{39}$ Outras amostras de esponjas deste gênero foram coletadas no litoral da Baía de Todos os Santos, ${ }^{11}$ dentre as quais uma amostra da esponja Dysidea sp.. O extrato MeOH desta esponja (BA99ES-38) apresentou atividade antibiótica contra 4 linhagens de Staphylococcus aureus resistente à oxacilina e contra $M$. tuberculosis H37Rv, bem como atividade citotóxica sobre a linhagem de células de câncer de cólon HCT-8. ${ }^{11}$

O fracionamento do extrato $\mathrm{MeOH}$ de Dysidea sp. por partição líquido-líquido e por uma série de separações cromatográficas (ver Parte Experimental) levou ao isolamento da lactona da furodisinina (14) e ao esterol $9 \alpha, 11 \alpha$-epoxicolest-7-eno-3 $\beta, 5 \alpha, 6 \alpha, 10$-tetrol-6acetato (15). A lactona da furodisinina $\mathbf{1 4}$ foi originalmente isolada da esponja Dysidea etheria..$^{40} \mathrm{O}$ esterol altamente oxigenado $\mathbf{1 5}$ foi isolado de outra esponja Dysidea sp. da ilha de Guam e teve sua estrutura completamente estabelecida por Gunasekera e Schmitz em $1983,{ }^{41}$ tendo sido reisolado como substância inédita em 2005 por 
<smiles>COC1=C(Br)C2=C(Br)C(Br)=C[C@@]2(O)CC(=O)O1</smiles><smiles>COc1c(Br)cc(CCN(C)C)cc1Br</smiles><smiles>[R]OC1=NO[C@@]2(C=C(Br)C(OC)=C(Br)[C@@H]2O)C1</smiles>

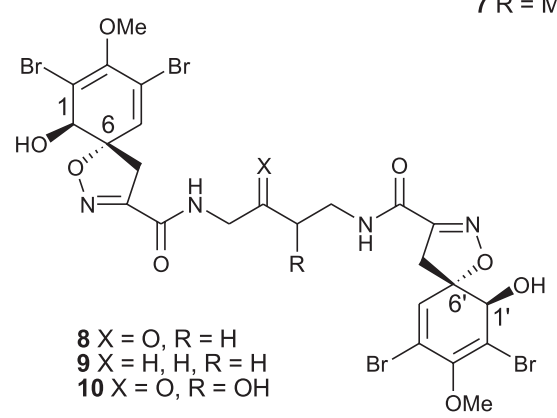

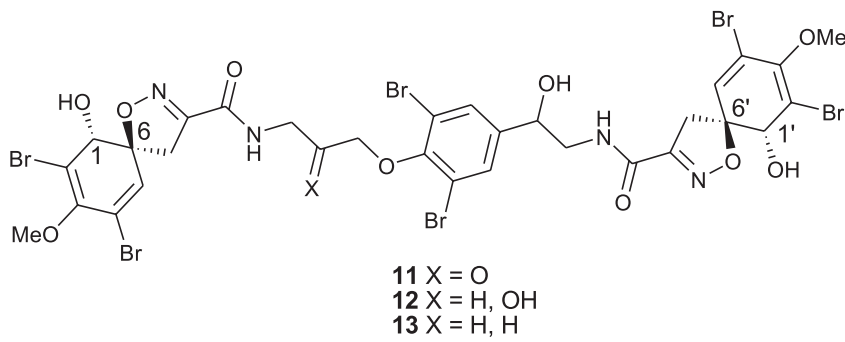

Huang e colaboradores a partir de uma esponja do gênero Dysidea do Mar da China. ${ }^{42} \mathrm{O}$ composto $\mathbf{1 5}$ apresentou atividade antifúngica contra Candida albicans, inibindo a bomba de efluxo de fluconazol, ${ }^{43}$ porém se mostrou inativo nos testes de atividade antituberculose contra $M$. tuberculosis $\mathrm{H} 37 \mathrm{Rv}$ por nós realizados.

O sesquiterpeno 14 teve sua estrutura elucidada como sendo a lactona da furodisinina, pela análise de seus dados espectroscópicos e espectrométricos (EMAR, RMN $-{ }^{1} \mathrm{H}, \mathrm{RMN}-{ }^{13} \mathrm{C}, \mathrm{HSQC}, \mathrm{COSY}$ ${ }^{1} \mathrm{H}-{ }^{1} \mathrm{H}$ e ROESY) e pela comparação de seus dados espectroscópicos com dados da literatura. ${ }^{40}$ Como discutido por Grode e Cardellina, ${ }^{40}$ a distinção entre a lactona da furodisinina (14) e a lactona da furodisina (16) não é trivial, e requer uma cuidadosa análise dos dados espectroscópicos. Os dados de $\mathbf{1 4}$ concordam de maneira excelente com os dados da literatura para a lactona da furodisinina,${ }^{40} \mathrm{e}$ diferem dos dados reportados para a lactona da furodisina (16) ${ }^{43}$ Infelizmente a pequena quantidade isolada do composto 14 não nos permitiu sua avaliação no bioensaio de atividade antituberculose.

Em conclusão, o presente trabalho apresenta o primeiro isolamento de derivados da dibromotirosina da esponja Aplysina fistularis, dos<smiles>CC1=C[C@H]2C[C@@]3(O)OC(=O)C=C3[C@@H](C)[C@H]2CC1</smiles>

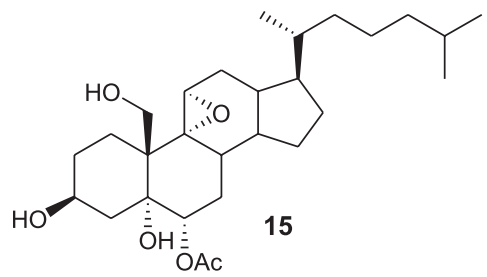<smiles>CC1=C[C@H]2CC3=CC(=O)O[C@@]3(O)[C@@H](C)[C@@H]2CC1</smiles>

quais a 11-ceto-fistularina-3 (11) apresentou atividade antituberculose com excelente índice de seletividade. Além disso, a investigação química do extrato ativo contra M. tuberculosis da esponja Dysidea sp., também realizado pela primeira vez, levou-nos a obter a lactona da furodisinina (14) e o esterol altamente oxigenado 15, dos quais o último mostrou ser inativo no teste de atividade antituberculose.

\section{Material suplementar}

Espectros de $\mathrm{RMN}-{ }^{1} \mathrm{H}$ e $\mathrm{RMN}-{ }^{13} \mathrm{C}$ dos compostos aeroplisinina-2 (4), 2-(3,5-dibromo-4-metoxifenil)- $N, N, N$-trimetiletanamônio (5), ácido verongidico (6), éster metílico do ácido verongidoico (7), 11-oxoaerotionina $(\mathbf{8})$, aerotionina $(\mathbf{9}), 11$-ceto-12-hidroxiaerotionina (10), 11-cetofistularina-3 (11) e fistularina-3 (12), estão disponíveis em http://quimicanova.sbq.org.br, na forma de arquivo PDF, com acesso livre.

\section{PARTE EXPERIMENTAL}

\section{Generalidades}

Os espectros na região do infravermelho foram obtidos em um aparelho FT-IR Bomem MB102 em pastilha de silício. Para as medidas de rotação específica foi usado um polarímetro 241 Perkin-Elmer. Os espectros na região do ultravioleta foram obtidos em $\mathrm{MeOH}$, em um aparelho Hitachi U-3210. Os espectros de RMN foram obtidos em DMSO- $d_{6}, \mathrm{MeOH}-d_{4}, \mathrm{MeCN}-d_{3}$ ou $\mathrm{CDCl}_{3}$, a $25^{\circ} \mathrm{C}$ utilizando TMS como padrão interno, com um aparelho Bruker ARX 9.4 Tesla operando em 400,35 MHz $\left({ }^{1} \mathrm{H}\right)$ e $100,10 \mathrm{MHz}\left({ }^{13} \mathrm{C}\right)$. Os espectros de massas foram obtidos utilizando dois equipamentos: espectrômetro de massas VG-7070 operando a uma voltagem de aceleração nominal de $70 \mathrm{eV}$, no qual os espectros obtidos por EI, FAB ou CI foram calibrados com perfluoroquerosene, álcool 3-nitrobenzílico ou polietilenoglicol; equipamento Hybrid Kratos Concept IIHQ, em modo FAB. Solventes de grau analítico (P.A.) foram utilizados para a extração e cromatografia em coluna de baixa pressãoflash. Solventes de grau cromatográfico (HPLC) e água MilliQ foram utilizados em separações por cromatografia líquida, usando um equipamento Waters de bomba quaternária 600, detector de duplo feixe UV 2487 e processador-registrador 746 . Vouchers da espécies de esponjas coletadas foram depositados na coleção de poríferos do Museu Nacional, Universidade Federal do Rio de Janeiro, Rio de Janeiro, RJ.

\section{Ensaio da atividade antimicobacteriana in vitro}

O procedimento deste bioensaio foi realizado exatamente como descrito anteriormente. ${ }^{24}$

\section{Estudo químico da esponja Aplysina fistularis}

A esponja Aplysina fistularis $(534,5 \mathrm{~g}$ ) foi coletada em seu habitat natural, na Baía de Todos os Santos, Salvador-BA, em 1999, e imediatamente congelada. Um espécimen padrão (MNRJ 2666) foi depositado no Museu Nacional, UFRJ. O material congelado foi liofilizado e mantido em EtOH 93\% a $-20{ }^{\circ} \mathrm{C}$. Quando da extração, o EtOH de preservação foi filtrado e o animal foi re-extraído com $\mathrm{MeOH}$ (triturado em liquidificador). $\mathrm{O}$ extrato $\mathrm{MeOH}$ foi filtrado, combinado com o EtOH de preservação e o extrato alcoólico foi evaporado até se obter uma suspensão aquosa. Esta foi diluída com $\mathrm{MeOH}$ de maneira a se obter uma solução de composição aproximada $\mathrm{MeOH}-\mathrm{H}_{2} \mathrm{O}$ 9:1. Esta solução $(500 \mathrm{~mL})$ foi particionada com $n$-hexano (3 x $500 \mathrm{~mL})$. A fração $\mathrm{MeOH}-\mathrm{H}_{2} \mathrm{O}$ foi então evaporada, diluída em $\mathrm{H}_{2} \mathrm{O}(500 \mathrm{~mL})$ particionada com AcOEt $(3 \times 500 \mathrm{~mL})$. 
A fração AcOEt foi evaporada até a secura. Foram obtidos 2,2 $\mathrm{g}$ do extrato $n$-hexano e 5,5 g do extrato AcOEt. A fração aquosa foi evaporada até a secura, da qual se obteve $11,7 \mathrm{~g}$.

O extrato AcOEt foi submetido a uma separação por cromatografia de permeação em gel de Sephadex LH-20 (50 g, 170 x 2 cm), utilizando-se $\mathrm{MeOH}$ como eluente. Foram obtidas 7 frações, AfisAcOEt-A (274 mg), Afis-AcOEt-B (172 mg), Afis-AcOEt-C (37 mg), Afis-AcOEt-D (295 mg), Afis-AcOEt-E/F (234 mg), Afis-AcOEt-G $(686 \mathrm{mg})$ e Afis-AcOEt-H $(171 \mathrm{mg})$. A fração Afis-AcOEt-A não apresentou derivados de dibromotirosina.

A fração Afis-AcOEt-B foi submetida a uma separação por cromatografia em coluna de sílica gel derivatizada com grupos cianopropila (5 g), utilizando-se um gradiente de AcOEt/MeOH 1:1 em $\mathrm{CH}_{2} \mathrm{Cl}_{2}(10: 90,20: 80,30: 70,40: 60,50: 50$ e 100\% AcOEt/MeOH 1:1). Após a análise por cromatografia em camada delgada (CCD) das 36 frações obtidas (eluente: $\mathrm{CH}_{2} \mathrm{Cl}_{2} / \mathrm{MeOH}$ 9:1; revelador: $\mathrm{UV}$, $254 \mathrm{~nm}$ ), estas foram reunidas em frações: Afis-AcOEt-B1 (104 mg), Afis-AcOEt-B2 (15 mg) e Afis-AcOEt-B3 (23 mg). A fração Afis-AcOEt-B1 foi submetida a uma separação por cromatografia em coluna de sílica gel derivatizada com grupos cianopropila $(5 \mathrm{~g})$, utilizando-se um gradiente de $\mathrm{AcOEt} / \mathrm{MeOH}$ 8:2 em $\mathrm{CH}_{2} \mathrm{Cl}_{2}$ (10:90, 20:80, 30:70, 40:60, 50:50, 100\% AcOEt/MeOH 8:2). A análise por CCD (eluente: $\mathrm{CH}_{2} \mathrm{Cl}_{2} / \mathrm{MeOH}$ 9:1; revelador: $\mathrm{UV}, 254 \mathrm{~nm}$ ) permitiu reunir as 42 frações obtidas em 3 frações: Afis-AcOEt-B1A (46 mg), Afis-AcOEt-B1B (8 mg) e Afis-AcOEt-B1C/D (14 mg). A fração Afis-AcOEt-B1B mostrou estar pura e foi identificada como sendo o 2-(3,5-dibromo-4-metoxifenil)- $N, N, N$-trimetiletanamônio (5). ${ }^{16,25}$

A fração Afis-AcOEt-C foi submetida a uma separação por cromatografia em coluna de sílica gel derivatizada com grupos octadecila $\left(\mathrm{C}_{18}, 5 \mathrm{~g}\right)$, utilizando-se um gradiente de $\mathrm{MeOH}$ em $\mathrm{H}_{2} \mathrm{O}$ (10:90, 20:80, 30:70, 40:60, 50:50, 60:40, 70:30, 80:20, 90:10, 100\% MeOH). Após a análise por $\mathrm{CCD}$ (eluente: $\mathrm{CH}_{2} \mathrm{Cl}_{2} / \mathrm{MeOH}$ 9:1, revelador UV 250 $\mathrm{nm})$, as 100 frações obtidas foram reunidas em 5: Afis-AcOEt-C1 (22 mg), Afis-AcOEt-C2 (2 mg), Afis-AcOEt-C3 (11 mg), AfisAcOEt-C4 (11 mg) e Afis-AcOEt-C5 (3 mg). Todavia, análises por $\mathrm{RMN}-{ }^{-1} \mathrm{H}$ indicaram que nenhuma destas frações apresentou derivados da dibromotirosina.

A fração Afis-AcOEt-D foi submetida a uma separação por cromatografia em coluna de sílica gel derivatizada com grupos cianopropila (5 g), utilizando-se um gradiente de A (AcOEt/MeOH 9:1) em B $\left(\mathrm{CH}_{2} \mathrm{Cl}_{2}\right.$ /éter de petróleo 8:2). As proporções de A e B foram de 10:90, 20:80, 30:70, 40:60, 50:50, 100\% A. Foram obtidas 144 frações, as quais, após análise por $\mathrm{CCD}$ (eluente: $\mathrm{CH}_{2} \mathrm{Cl}_{2} / \mathrm{MeOH}$ 9:1; revelador: UV $254 \mathrm{~nm}$ ), puderam ser reunidas em 4 frações: Afis-AcOEt-D1 (28 mg), Afis-AcOEt-D2 (84 mg), Afis-AcOEt-D3 (21 mg), Afis-AcOEt-D4 (47 mg). Análises por RMN- ${ }^{1} \mathrm{H}$ das frações obtidas não indicaram a presença de derivados da dibromotirosina.

A fração Afis-AcOEt-E/F foi submetida a uma separação por cromatografia em coluna de sílica gel em modo "relâmpago", utilizando-se um gradiente de A ( $\mathrm{AcOEt} / \mathrm{MeOH}$ 95:5) em B $\left(\mathrm{CH}_{2} \mathrm{Cl}_{2}\right.$ / éter de petróleo 8:2). As proporções utilizadas de $\mathrm{A}$ e $\mathrm{B}$ foram de 10:90, 20:80, 30:70, 40:60, 50:50, 100\% A. Foram obtidas 93 frações as quais, após análise por $\mathrm{CCD}$ (eluente: $\mathrm{CH}_{2} \mathrm{Cl}_{2} / \mathrm{MeOH}$; revelador UV $254 \mathrm{~nm}$ ) puderam ser reunidas em 8 frações: Afis-AcOEt-E/F1 (36 mg), Afis-AcOEt-E/F2/3 (25 mg), Afis-AcOEt-E/F4 (3,5 mg), Afis-AcOEt-E/F5 (40 mg), Afis-AcOEt-E/F6 (15 mg), Afis-AcOEtE/F7 (20 mg), Afis-AcOEt-E/F8/9 (79 mg) e Afis-AcOEt-E/F10 (10 $\mathrm{mg}$ ). A fração Afis-AcOEt-E/F8/9 foi submetida a uma separação por cromatografia em coluna de sílica gel derivatizada com grupos octadecila $\left(\mathrm{C}_{18}, 2 \mathrm{~g}\right)$, utilizando-se um gradiente de $\mathrm{MeOH}$ em $\mathrm{H}_{2} \mathrm{O}$ (proporções: $\mathrm{MeOH} / \mathrm{H}_{2} \mathrm{O}$ 10:90, 20:80, 30:70, 40:60, 50:50, 75:25, $100 \% \mathrm{MeOH}$ ). Após a análise por $\mathrm{CCD}$ (eluente: $\mathrm{CH}_{2} \mathrm{Cl}_{2} / \mathrm{MeOH}$ 9:1; revelador: UV $254 \mathrm{~nm}$ ), as 40 frações obtidas foram reunidas em 3 frações: Afis-AcOEt-E/F8/9A (22 mg), Afis-AcOEt-E/F8/9B (15 $\mathrm{mg}$ ) e Afis-AcOEt-E/F8/9C (30 mg). A fração Afis-AcOEt-E/F2/3 foi purificada por CLAE (coluna: Waters Phenyl $\mu$ Bondapak 7,8 x $300 \mathrm{~mm}$; fluxo: $1,0 \mathrm{~mL} / \mathrm{min}$; detector: UV $\lambda_{\text {max }} 254 \mathrm{~nm}$; eluente: $\mathrm{MeOH} / \mathrm{H}_{2} \mathrm{O}$ 8:2) e forneceu o éster metílico do ácido verongidoico $(\mathbf{7}, 2 \mathrm{mg})^{18}$ e a aeroplisinina-2 (4, $\left.4 \mathrm{mg}\right) \cdot{ }^{15,24}$

A fração Afis-AcOEt-G foi submetida a uma separação por cromatografia em coluna de sílica gel "relâmpago" (30 g), utilizandose um gradiente de $\mathrm{A}$ (AcOEt/MeOH 95:5) em $\mathrm{B}\left(\mathrm{CH}_{2} \mathrm{Cl}_{2} /\right.$ éter de petróleo 8:2), nas seguintes proporções A/B: 10/90, 20/80, 30/70, 40/60, 50/50, 100\% A. Foram obtidas 98 frações que foram analisadas por CCD (eluente: $\mathrm{CH}_{2} \mathrm{Cl}_{2} / \mathrm{MeOH} 9: 1$; revelador: UV $254 \mathrm{~nm}$ ) e reunidas em 7 frações: Afis-AcOEt-G1 (7 mg), Afis-AcOEt-G2 (6 $\mathrm{mg}$ ), Afis-AcOEt-G3 (3 mg), Afis-AcOEt-G4 (15 mg), Afis-AcOEtG5 (272 mg), Afis-AcOEt-G6 (152 mg), Afis-AcOEt-G7 (217 mg). A fração Afis-AcOEt-G5 foi separada por CLAE (coluna: Waters Phenyl $\mu$ Bondapak 7,8 x $300 \mathrm{~mm}$; fluxo: $1,0 \mathrm{~mL} / \mathrm{min}$; detector: UV $\lambda_{\max } 254 \mathrm{~nm}$; eluente: $\mathrm{MeOH} / \mathrm{H}_{2} \mathrm{O}$ 7:3). A fração Afis-AcOEt-G5D $(80 \mathrm{mg})$ foi identificata como sendo a fistularina-3 (12). ${ }^{12} \mathrm{~A}$ fração Afis-AcOEt-G5E (28 mg) foi identificada como sendo a 11-cetofistularina-3 (11). ${ }^{22}$ A fração Afis-AcOEt-G6 foi separada por CLAE (coluna: Phenomenex $\mathrm{C}_{18}, 19$ x $300 \mathrm{~mm}$; fluxo: 2,0 mL/min; detector: $\mathrm{UV} \lambda_{\max } 254 \mathrm{~nm}$; eluente: $\mathrm{MeOH} / \mathrm{H}_{2} \mathrm{O}$ 9:1). A terceira fração desta separação, Afis-AcOEt-G6C (121,2 mg) foi submetida a uma repurificação por CLAE (coluna: Waters Phenyl $\mu$ Bondapak de 7,8 x 300 $\mathrm{mm}$; fluxo: $1,0 \mathrm{~mL} / \mathrm{min}$; detector: UV $\lambda_{\text {max }} 254 \mathrm{~nm}$; eluente: $\mathrm{MeOH} /$ $\left.\mathrm{H}_{2} \mathrm{O} 55: 45\right)$. A fração Afis-AcOEt-G6C3 (1,4 mg) foi identificada como sendo o ácido verongidoico (6).17 A fração Afis-AcOEt-G6C6 (15 mg) foi identificada como sendo a 11-oxo-12-hidroxiaerotionina (10). ${ }^{21}$ A fração Afis-AcOEt-G6C7 (18 mg) foi identificada como sendo a 11-oxoaerotionina (8). ${ }^{19}$ A fração Afis-AcOEt-G6C8 (4 mg) foi identificada como sendo a aerotionina (9)..$^{20,21}$

A fração Afis-AcOEt-H foi submetida a uma separação por cromatografia em coluna de sílica gel "relâmpago" (30 g), utilizandose um gradiente de A (AcOEt/MeOH 95:5) em B $\left(\mathrm{CH}_{2} \mathrm{Cl}_{2} /\right.$ éter de petróleo 8:2), nas seguintes proporções A/B: 10/90, 20/80, 30/70, 40/60, 50/50, 100\% A. Foram obtidas 88 frações que puderam ser reunidas em 3 após análise por $\mathrm{CCD}$ (eluente: $\mathrm{CH}_{2} \mathrm{Cl}_{2} / \mathrm{MeOH}$ 9:1; revelador: UV $254 \mathrm{~nm})$ : Afis-AcOEt-H1 (15 mg), Afis-AcOEt-H2 (58 mg), Afis-AcOEt-H3 (34 mg). Análises por RMN- ${ }^{1} \mathrm{H}$ das frações obtidas não indicaram a presença de derivados da dibromotirosina.

\section{Estudo químico da esponja Dysidea sp.}

A esponja Dysidea sp., coletada na Baía de Todos os Santos, Salvador-BA em 1999, e foi imediatamente congelada após a coleta. Um espécimen padrão (MNRJ 2480) foi depositado no Museu Nacional, UFRJ. A esponja foi liofilizada e submetida a três extrações com $\mathrm{MeOH}$ após trituração em liquidificador. $\mathrm{O}$ extrato $\mathrm{MeOH}$ foi filtrado e parcialmente evaporado até um volume final de $400 \mathrm{~mL}$. A suspensão hidroalcoólica foi particionada com $n$-hexano $(3$ x $400 \mathrm{~mL})$. A fração hexânica (Dv-hex) foi evaporada até a secura e forneceu 1,6 g. A fração $\mathrm{MeOH} / \mathrm{H}_{2} \mathrm{O}$ foi evaporada até a total remoção do $\mathrm{MeOH}$. A suspensão aquosa obtida foi diluída com $\mathrm{H}_{2} \mathrm{O}$ até um volume final de $400 \mathrm{~mL}$. Esta suspensão foi particionada com AcOEt. Após evaporação das frações AcOEt e $\mathrm{H}_{2} \mathrm{O}$ obtiveram-se os extratos Dv-AcOEt $(1,2 \mathrm{~g})$ e Dv-Aq $(6,1 \mathrm{~g})$. Após análise por CCD (eluentes: $\mathrm{CH}_{2} \mathrm{Cl}_{2} /$ MeOH 9:1, 8:2 e 7:3; reveladores: UV, ninidrina e Dragendorff) a fração $\mathrm{H}_{2} \mathrm{O}$ foi descartada por não apresentar compostos de interesse.

O extrato Dv-hex (1,6 g) foi submetido a uma separação por cromatografia em coluna de sílica gel (10 g), utilizando-se como eluente um gradiente de AcOEt em $n$-hexano (10:90, 20:80, 30:70, 40:60, 50:50, 60:40, 70:30, 80:20, 90:10, 100\% AcOEt). Foram 
obtidas 11 frações diferentes: Dv-hex-1 (0,18 g), Dv-hex-2 (0,11 g), Dv-hex-3 (0,3 g), Dv-hex-4 (0,17 g), Dv-hex-5 (0,18 g), Dv-hex-6 (0,16 g), Dv-hex-7 (0,03 g), Dv-hex-8 (0,05 g), Dv-hex-9 (0,03 g), Dv-hex-10 (0,4 g) e Dv-hex-11 (0,01 g). As frações Dv-hex-5 e Dvhex-6 apresentaram compostos com intensa absorção no UV em placa de CCD (eluente: $n$-hexano/AcOEt 7:3), razão pela qual o estudo destas frações foi priorizado.

A fração Dv-hex-5 foi submetida a uma cromatografia em coluna pré-empacotada de sílica gel ( $2 \mathrm{~g}$ ) utilizando-se como eluente um gradiente de AcOEt (solvente A) em $n$-hexano/ $\mathrm{CH}_{2} \mathrm{Cl}_{2} 1: 1$ (solvente B) nas proporções A/B 10/90, 20/80, 30/70, 40/60, 50/50, 100\% A e $100 \% \mathrm{MeOH}$. Após análise por CCD das 60 frações obtidas (eluente: $n$-hexano/AcOEt 7:3; revelador: UV $254 \mathrm{~nm}$ e spray de ácido fosfomolíbdico, seguido de aquecimento em placa a cerca de $80^{\circ} \mathrm{C}$ ) estas foram reunidas em 8 frações, denominadas Dv-hex-5A a Dv-hex-5F, Dv-hex-5AcOEt (eluída com AcOEt 100\%) e Dv-hex$5 \mathrm{MeOH}$ (eluída com MeOH 100\%). A fração Dv-hex-5B (0,011 g) apresentou composto majoritário ainda impuro, e foi purificada por CLAE (coluna: Waters $\mu$-Porasil sílica gel $5 \mu \mathrm{m}, 19$ x 300 mm; eluente: $n$-hexano/AcOEt/MeOH 80:18:2; fluxo: $1 \mathrm{~mL} / \mathrm{min}$; detecção: UV $\lambda_{\text {max }} 254 \mathrm{~nm}$ ). Obteve-se 1,4 mg da lactona da furodisinina (14). ${ }^{40}$ As outras frações obtidas do extrato hexânico apresentaram somente esteróis, esteroides, ésteres do glicerol e ácidos graxos e não foram investigadas.

A fração Dv-AcOEt (1,2 g) foi filtrada para eliminação de sais inorgânicos e subsequentemente submetida a uma cromatografia em coluna pré-empacotada de sílica gel derivatizada com grupos cianopropila (5 g), utilizando-se como eluente um gradiente de AcOEt/MeOH 1:1 (solvente $\mathrm{A}$ ) em $\mathrm{CH}_{2} \mathrm{Cl}_{2}$ (solvente $\mathrm{B}$ ) nas seguintes proporções A/B: 10/90, 20/80, 30/70, 40/60, 50/50, 100\% A. As 60 frações obtidas foram analisadas por CCD (eluentes: $n$-hexano/AcOEt 7:3 ou 1:1; reveladores: UV $254 \mathrm{~nm}$ e e spray de ácido fosfomolíbdico, seguido de aquecimento em placa a cerca de $\left.80^{\circ} \mathrm{C}\right)$, e reunidas em quatro frações: Dv-AcOEt-1 (0,47 g), Dv-AcOEt-2 (0,034 g), Dv-AcOEt-3 (0,034 g) e Dv-AcOEt-4 (0,006 g). A fração Dv-AcOEt-1 foi submetida a uma separação por cromatografia em coluna de Sephadex LH-20 (eluente: $\mathrm{MeOH}$ ), da qual se obteve 7 frações: Dv-AcOEt-1A a Dv-AcOEt-1G. Devido à sua similaridade cromatográfica, as frações Dv-AcOEt$1 \mathrm{C}(0,15 \mathrm{~g})$ e Dv-AcOEt-1D $(0,11 \mathrm{~g})$ foram reunidas, originando a fração Dv-AcOEt-1C/D (0,26 g). Esta foi submetida a uma nova separação cromatográfica, em coluna de sílica gel derivatizada com grupos cianopropila (5 g), utilizando-se um eluente de AcOEt/MeOH 75:25 (solvente $\mathrm{A}$ ) em $\mathrm{CH}_{2} \mathrm{Cl}_{2}$ (solvente $\mathrm{B}$ ), nas seguintes proporções A/B: 10/90, 20/80, 30/70, 40/60, 50/50, 100\% A. Foram obtidas 50 frações que, após análises por CCD (eluentes: $n$-hexano/AcOEt 7:3 ou 1:1; reveladores: UV $254 \mathrm{~nm}$ e e spray de ácido fosfomolíbdico, seguido de aquecimento em placa a cerca de $80{ }^{\circ} \mathrm{C}$ ) foram reunidas em 3 frações, das quais a primeira, Dv-AcOEt-1C/D1 (0,19 g), foi submetida a uma nova separação em coluna de sílica gel derivatizada com grupos cianopropila (2 g), utilizando-se um gradiente de AcOEt/ MeOH 9:1 (solvente A) em $\mathrm{CH}_{2} \mathrm{Cl}_{2} / n$-hexano 9:1 (solvente B), nas seguintes proporções A/B: 10/90, 20/80, 30/70, 40/60, 50/50, 100\% A. Após análises por CCD (eluentes: $n$-hexano/AcOEt 7:3 ou 1:1; reveladores: UV $254 \mathrm{~nm}$ e e spray de ácido fosfomolíbdico, seguido de aquecimento em placa a cerca de $80{ }^{\circ} \mathrm{C}$ ) as 47 frações obtidas foram reunidas em 5 frações, das quais a segunda, Dv-AcOEt-1C/ D1A $(0,14 \mathrm{~g})$, foi novamente separada, desta vez por cromatografia em coluna de sílica gel ( $2 \mathrm{~g}$ ) utilizando-se um gradiente de AcOEt/ MeOH 95:5 (solvente A) em $n$-hexano/ $\mathrm{CH}_{2} \mathrm{Cl}_{2}$ 1:1 (solvente B), nas seguintes proporções A/B: 10:90, 20:80, 30:70, 40:60, 50:50, 100\% A. Análises por CCD das frações obtidas (eluentes: $n$-hexano/AcOEt 7:3 ou 1:1; reveladores: UV $254 \mathrm{~nm}$ e e spray de ácido fosfomolíbdico, seguido de aquecimento em placa a cerca de $80{ }^{\circ} \mathrm{C}$ ) levaram à reunião das 38 frações em 5, das quais a segunda, Dv-AcOEt-1C/ D1A2 (0,047 g), apresentou solubilidade parcial em MeOH. Após filtração, a fração insolúvel $(0,035 \mathrm{~g})$ foi solubilizada em $0,5 \mathrm{~mL}$ de DMSO e purificada por CLAE (coluna: inertsil ODS-3 (GL Sciences, Inc) $\mathrm{C}_{18}$, dimensões 250 x 4,6 mm; $5 \mu \mathrm{m}$; eluente: $\mathrm{MeOH} / \mathrm{H}_{2} \mathrm{O}$ 7:3; fluxo $1 \mathrm{~mL} / \mathrm{min}$; detecção: UV $\lambda_{\max } 254 \mathrm{~nm}$ ). As frações obtidas desta separação ainda mostraram ser misturas. A fração obtida da lavagem da coluna com $\mathrm{MeOH} 100 \%$ resultou na obtenção de $1,8 \mathrm{mg}$ do $9 \alpha, 11 \alpha$-epoxicolest-7-eno-3 $\beta, 5 \alpha, 6 \alpha, 10$-tetrol-6-acetato (15). ${ }^{41,42}$

\section{AGRADECIMENTOS}

À gentileza do Prof. B. Copp (University of Auckland, Auckland, Nova Zelândia), do Dr. D. E. Williams e Prof. R. J. Andersen (University of British Columbia, Vancouver, Canadá) pela ajuda na obtenção dos espectros de massas de baixa e alta resolução, bem como dos espectros de RMN a $600 \mathrm{MHz}$. O apoio financeiro para a realização deste trabalho foi concedido pela American Society of Pharmacognosy Foundation (1998 Research Starter Grant) e pela Fundação de Amparo à Pesquisa do Estado de São Paulo (05/60175-2) a R. G. S. Berlinck, no programa BIOprospecTA/BIOTA. Renata C. Gandolfi e Marina B. Medina agradecem ao programa PIBIC/CNPq/USP pelas bolsas de iniciação científica concedidas. Simone P. de Lira (06/60510-9) agradece à FAPESP pela bolsas de pós-doutorado concedida. R. G. S. Berlinck, E. Hajdu, e A. G. Ferreira também agradecem ao CNPq pelas bolsas de produtividade em pesquisa.

\section{REFERÊNCIAS}

1. Riccardi, G.; Pasca, M. R.; Buroni, S.; Future Microbiol. 2009, 4, 597.

2. Smith, N. H.; Hewinson, R. G.; Kremer, K.; Brosch, R.; Gordon, S. V.; Nature Rev. Microbiol. 2009, 7, 537.

3. Sacchettini, J. C.; Rubin, E. J.; Freundlich, J. S.; Nature Rev. Microbiol. 2008, 6, 41 .

4. Copp, B. R.; Pearce, A. N.; Nat. Prod. Rep. 2007, 24, 278.

5. Laport, M. S.; Santos, O. C. S.; Muricy, G.; Curr. Pharm. Biotechnol. 2009, 10, 86 .

6. Palomino, J. C.; Ramos, D. F.; da Silva, P. A.; Curr. Med. Chem. 2009, 16, 1898.

7. Kishore, N.; Mishra, B. B.; Tripathi, V.; Tiwari, V. J.; Fitoterapia 2009, $80,149$.

8. Basso, L. A.; Silva, L. H. P.; Fett-Neto, A. G.; Junior, W. F. A.; Moreira, I. S.; Palma, M. S.; Calixto, J. B.; Filho, S. A.; Santos, R. R.; Soares, M. B. P.; Santos, D. S.; Mem. Inst. Oswaldo Cruz 2005, 100, 575.

9. McGawa, L. J.; Lall, N.; Meyer, J. J. M.; Eloff, J. N.; J. Ethnopharm. 2008, 119, 482 .

10. Wright, G. D.; Sutherland, A. D.; Trends Mol. Med. 2007, 13, 260.

11. Seleghim, M. H. R.; Lira, S. P.; Kossuga, M. H.; Batista, T.; Berlinck, R. G. S.; Hajdu, E.; Muricy, G.; Rocha, R. M.; Nascimento, G. F.; Silva, M.; Pimenta, E. F.; Thiemann, O. H.; Oliva, G.; Cavalcanti, B. C.; Pessoa, C.; Moraes, M. O.; Hajdu, E.; Peixinho, S.; Rocha, R. M.; Rev. Bras. Farmacogn. 2007, 17, 287.

12. Oliveira, M. F.; Oliveira, J. H. H. L.; Galetti, F. C. S.; Souza, A. O.; Silva, C. L.; Hajdu, E.; Peixinho, S.; Berlinck, R. G. S.; Planta Med. 2006, 72, 437 .

13. Oliveira, J. H. H. L.; Seleghim, M. H. R.; Timm, C.; Grube, A.; Köck, M.; Nascimento, G. G. F.; Martins, A. C. T.; Silva, E. G. O.; Souza, A. O.; Galetti, F. C. S.; Minarini, P. R. R.; Silva, C. L. L.; Hajdu, E.; Berlinck, R. G. S.; Mar. Drugs 2006, 4, 1.

14. de Oliveira, J. H. H. L.; Grube, A.; Kock, M.; Berlinck, R. G. S.; Macedo, M. L.; Ferreira, A. G.; Hajdu, E.; J. Nat. Prod. 2004, 67, 1685.

15. Minale, L.; Sodano, G.; Chan, W. R.; Chen, A. M.; J. Chem. Soc., Chem. Commun. 1972, 674. 
16. Ciminiello, P.; Fattorusso, E.; Magno, S.; Pansini, M.; J. Nat. Prod. 1994, 57, 1564.

17. Aiello, A.; Fattorusso, E.; Menna, M.; Pansini, M.; Biochem. Syst. Ecol. 1995, 23, 377

18. Ciminiello, P.; Fattorusso, E.; Magno, S.; Pansini, M.; J. Nat. Prod. 1994, 57, 1564.

19. Acosta, A. L.; Rodriguez, A. D.; J. Nat. Prod. 1992, 55, 1007.

20. Fattorusso, E.; Minale, L.; Sodano, G.; Moody, K.; Thomson, R. H.; J. Chem. Soc. D: Chem. Commun. 1970, 752

21. Ciminiello, P.; Costantino, V.; Fattorusso, E.; Magno, S.; Mangoni, A.; Pansini, M.; J. Nat. Prod. 1994, 57, 705.

22. Gunasekera, S. P.; Cross, S. S.; J. Nat. Prod. 1992, 55, 509.

23. Gopichand, Y.; Schmitz, F. J.; Tetrahedron Lett. 1979, 3921.

24. Kossuga, M. H.; Lira, S. P.; Nascimento, A. M.; Gambardella, M. T. P.; Berlinck, R. G. S.; Torres, Y. R.; Nascimento, G. G. F.; Pimenta, E. F.; Silva, M.; Thiemann, O. H.; Oliva, G.; Tempone, A. G.; Melhem, M. S. C.; Souza, A. O.; Galetti, F. C. S.; Silva, C. L.; Cavalcanti, B.; Pessoa, C. O.; Moraes, M. O.; Hajdu, E.; Peixinho, S.; Rocha, R. M.; Quim. Nova 2007, 30, 1194.

25. Granato, A. C.; Oliveira, J. H. H. L.; Seleghim, M. H. R.; Berlinck, R. G. S.; Macedo, M. L.; Ferreira, A. G.; Rocha, R. M.; Hajdu, E.; Peixinho, S.; Pessoa, C. O.; Moraes, M. O.; Cavalcanti, B. C.; Quim. Nova 2005, 28, 192.

26. Ciminiello, P.; Dell'Aversano, C.; Fattorusso, E.; Magno, S.; Pansini, M.; J. Nat. Prod. 2000, 63, 263.

27. Nuñez, C. V.; Almeida, E. V. R.; Granato, A. C.; Marques, S. O.; Santos, K. O.; Pereira, F. R.; Macedo, M. L.; Ferreira, A. G.; Hajdu, E.; Pinheiro, U. S.; Muricy, G.; Peixinho, S.; Freeman, C. J.; Gleason, D. F.; Berlinck, R. G. S.; Biochem. Syst. Ecol. 2008, 36, 283.

28. Mancini, I.; Guella, G.; Laboute, P.; Debitus, C.; Pietra, F.; J. Chem. Soc. Perkin Trans I 1993, 3121.

29. Rogers, E. W.; Oliveira, M. F.; Berlinck, R. G. S.; König, G. M.; Molinski, T. F.; J. Nat. Prod. 2005, 68, 891.
30. Saeki, B. M.; Granato, A. C.; Berlinck, R. G. S.; Magalhães, A.; Schefer, A. B.; Ferreira, A. G.; Pinheiro, U. G.; Hajdu, E.; J. Nat. Prod. 2002, 65, 796; Saeki, B. M.; Granato, A. C.; Berlinck, R. G. S.; Magalhães, A.; Schefer, A. B.; Ferreira, A. G.; Pinheiro, U. G.; Hajdu, E.; J. Nat. Prod. 2003, 66, 1039; Lira, T. O.; Nascimento, G. F. F.; Hajdu, E.; Berlinck, R. G. S.; J. Braz. Chem. Soc. 2006, 17, 1233.

31. McMillan, J. A.; Paul, I. C.; Goo, Y. M.; Rinehart, K. L.; Krueger, W. C.; Pschigoda, L. M.; Tetrahedron Lett. 1981, 22, 39.

32. Tymiak, A. A.; Rinehart, K. L.; J. Am. Chem. Soc. 1981, 103, 6763.

33. Carney, J. R.; Rinehart, K. L.; J. Nat. Prod. 1995, 58, 971.

34. Gulavita, N. K.; Pomponi, S. A.; Wright, A. E.; Garay, M.; Sills, M. A.; J. Nat. Prod. 1995, 58, 954.

35. Compagnone, R. S.; Avila, R.; Suarez, A. I.; Abrams, O. V.; Rangel, H. R.; Arvelo, F.; Pina, I. C.; Merentes, E.; J. Nat. Prod. 1999, 62, 1443.

36. Silva, C. L.; Berlinck, R. G. S.; Souza, A. O.; Oliveira, M. F.; P. I. 0.600.470-9.7., 2006

37. Blunt, J. W.; Copp, B. R.; Hu, W.-P.; Munro, M. H. G.; Northcote, P. T.; Prinsep, M. R.; Nat. Prod. Rep. 2009, 26, 170; Faulkner, D. J.; Nat. Prod. Rep. 2002, 19, 1.

38. Sharma G. M.; Vig, B.; Tetrahedron Lett. 1972, 17, 1715.

39. Marques, S. O.; Veloso, K.; Ferreira, A. G.; Hajdu, E.; Peixinho, S.; Berlinck, R. G. S.; Nat. Prod. Commun. 2009, 4, 917; Williams, D. E.; Marques, S. O.; Hajdu, E.; Peixinho, S.; Andersen, R. J; Berlinck, R. G. S.; J. Nat. Prod. 2009, 72, 1691.

40. Grode, S. H.; Cardellina II, J. H.; J. Nat. Prod. 1984, 47, 76; Dumdei, E.; Kubanek, J.; Coleman, J. E.; Pika, J.; Andersen, R. J.; Steiner, J. R.; Clardy, J.; Can. J. Chem. 1997, 75, 773.

41. Gunasekera, S. P.; Schmitz, F. J.; J. Org. Chem. 1983, 48, 885.

42. Huang, X.-C.; Guo, Y.-W.; Mollo, E.; Cimino, G.; Helv. Chim. Acta 2005, 88, 281.

43. Goetz, G. H.; Harrigan, G. G.; Likos, J.; J. Nat. Prod. 2001, 64, 1486. 


\section{METABÓLITOS SECUNDÁRIOS DAS ESPONJAS Aplysina fistularis E Dysidea sp. E ATIVIDADE}

\section{ANTITUBERCULOSE DA 11-CETOFISTULARINA-3}

Renata C. Gandolfi, Marina B. Medina e Roberto G. S. Berlinck*

Instituto de Química de São Carlos, Universidade de São Paulo, CP 780, 13560-970 São Carlos - SP, Brasil

Simone P. Lira

Departamento de Ciências Exatas, Escola Superior de Agricultura “Luiz Queiroz”, Universidade de São Paulo, CP 9, 13418-900

Piracicaba - SP, Brasil

Fabio Cícero de Sá Galetti e Célio L. Silva

Farmacore Biotecnologia Ltda, Ribeirão Preto - SP, / Faculdade de Medicina de Ribeirão Preto, Universidade de São Paulo, Av. Bandeirantes, 3900, 14049-900 Ribeirão Preto - SP, Brasil

Katyuscya Veloso e Antonio G. Ferreira

Departamento de Química, Universidade Federal de São Carlos, CP 676, 13560-970 São Carlos - SP, Brasil

Eduardo Hajdu

Museu Nacional, Universidade Federal do Rio de Janeiro, Quinta da Boa Vista, s/n, 20940-040 Rio de Janeiro - RJ, Brasil

Solange Peixinho

Departamento de Biologia, Universidade Federal da Bahia, Campus Univ. Ondina, 40170-290 Salvador - BA, Brasil

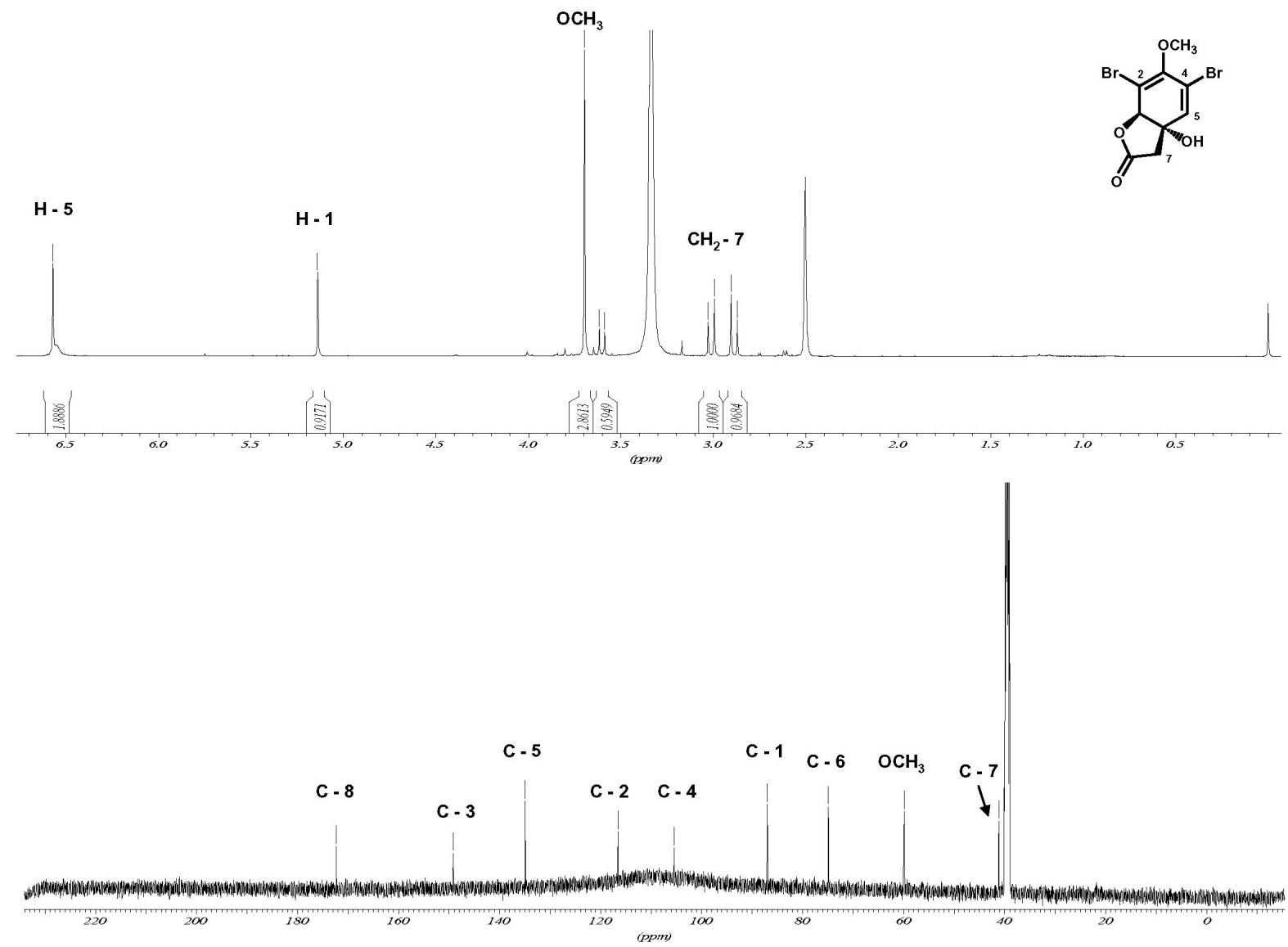

Figura 1S. Espectros de RMN - ${ }^{1} \mathrm{H}\left(\mathrm{DMSO}-\mathrm{d}_{6}, 400 \mathrm{MHz}\right)$ e $\mathrm{RMN}-{ }^{13} \mathrm{C}\left(\mathrm{DMSO}-\mathrm{d}_{6}, 100 \mathrm{MHz}\right)$ da aeroplisinina-2 (4)

*e-mail: rgsberlinck@iqsc.usp.br 


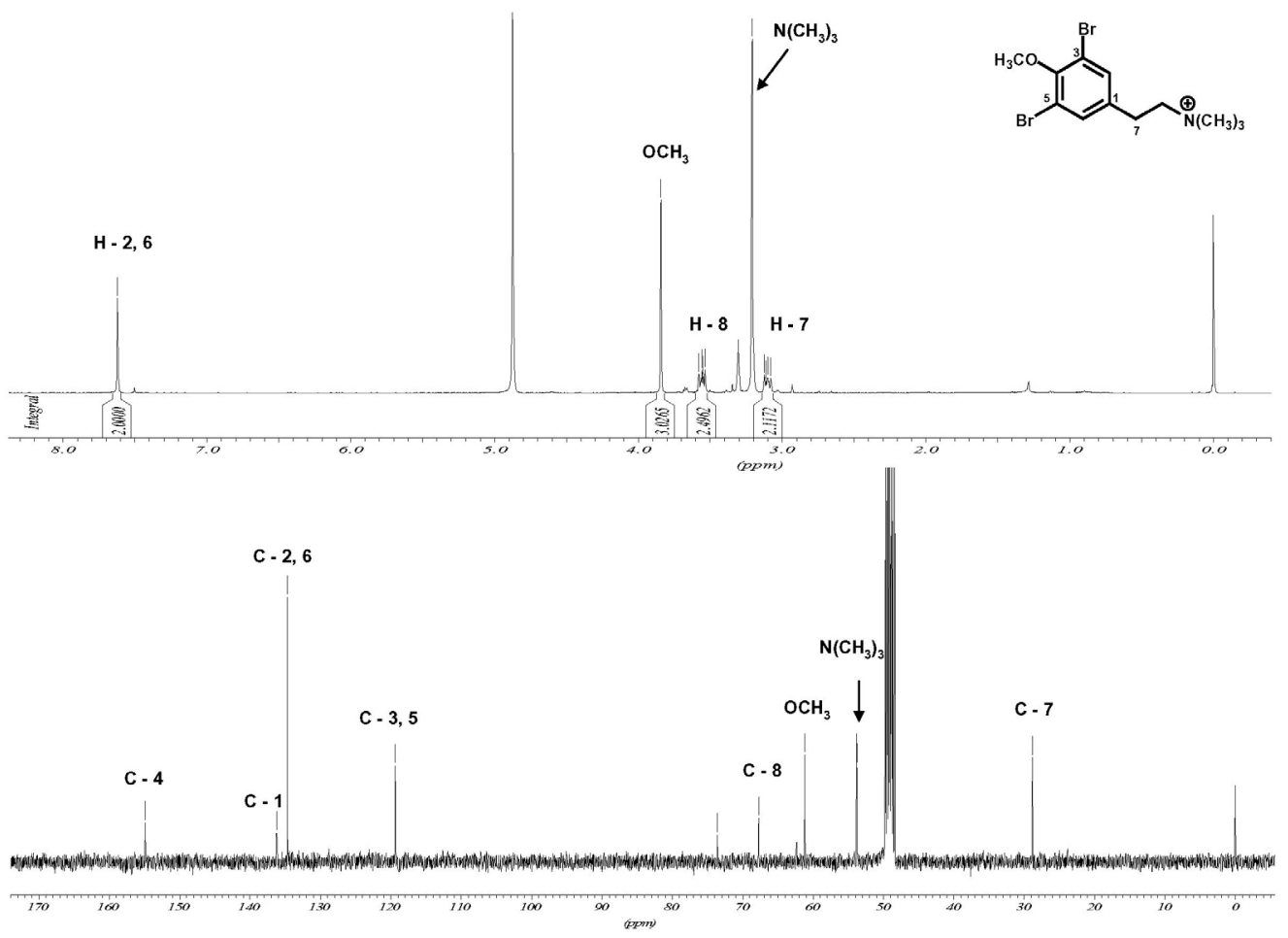

Figura 2S. Espectros de $\mathrm{RMN}-{ }^{1} \mathrm{H}\left(\mathrm{CD} \mathrm{O}_{3} \mathrm{OD}, 400 \mathrm{MHz}\right)$ e $\mathrm{RMN}-{ }^{13} \mathrm{C}\left(\mathrm{CD}_{3} \mathrm{OD}, 100 \mathrm{MHz}\right)$ do 2-(3,5-dibromo-4-metoxifenil)-N,N,N-trimetiletanamônio (5)
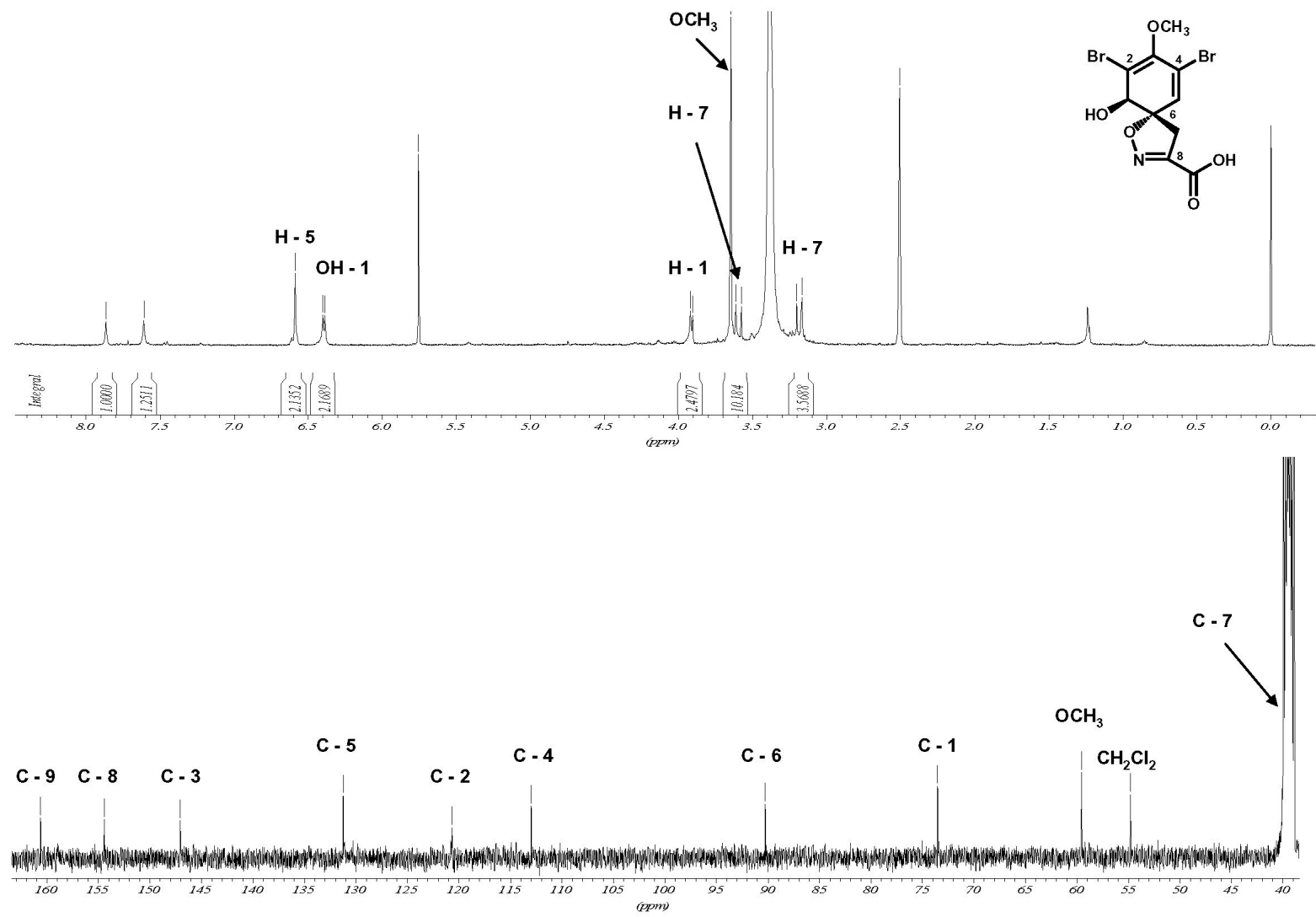

Figura 3S. Espectros de RMN $-{ }^{1} \mathrm{H}\left(\mathrm{DMSO}-\mathrm{d}_{6}, 400 \mathrm{MHz}\right)$ e RMN $-{ }^{13} \mathrm{C}\left(\mathrm{DMSO}-\mathrm{d}_{6}, 100 \mathrm{MHz}\right)$ do do ácido verongidóico (6) 

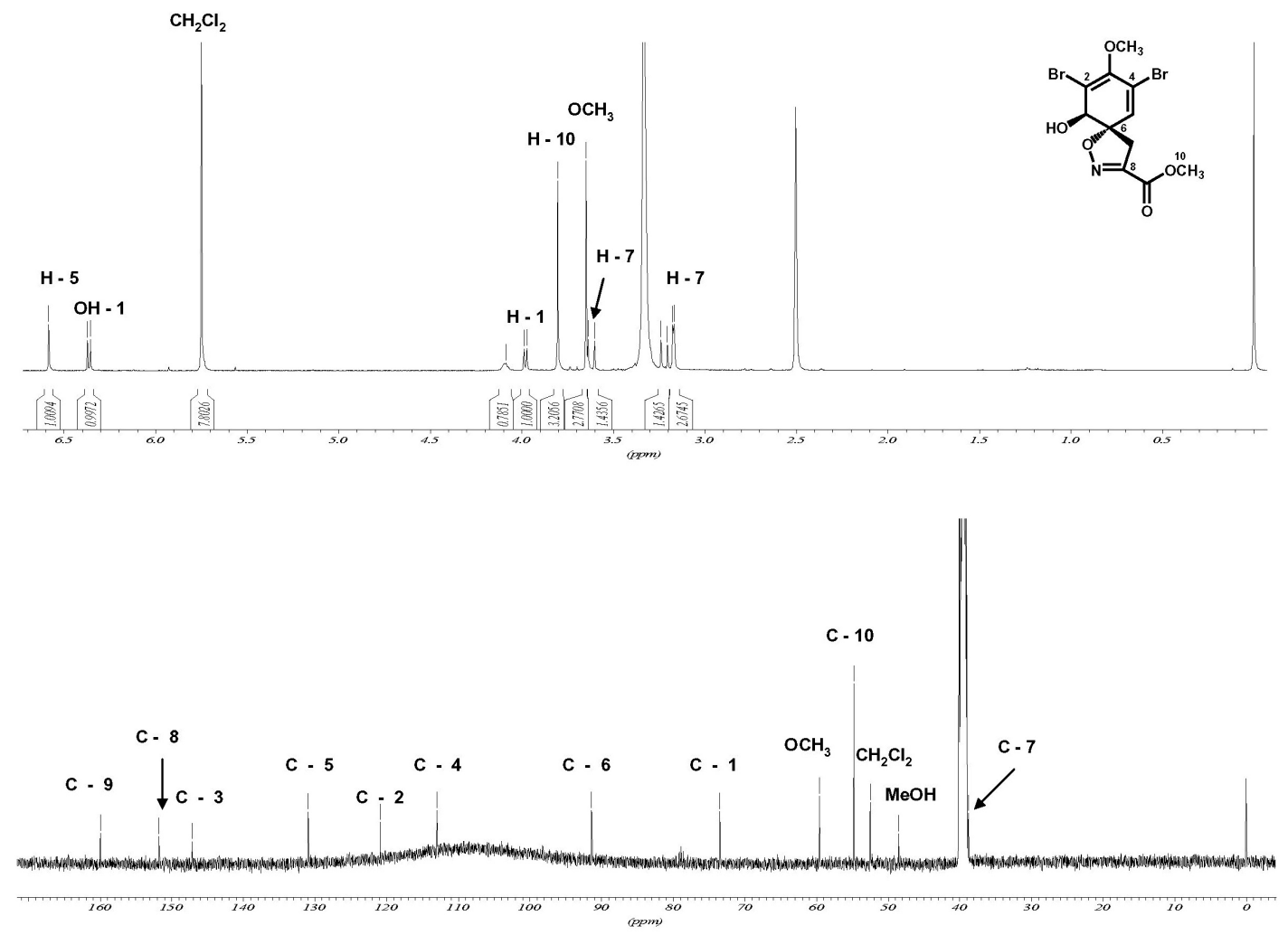

Figura 4S. Espectros de RMN $-{ }^{1} \mathrm{H}\left(\mathrm{DMSO}-\mathrm{d}_{6}, 400 \mathrm{MHz}\right)$ e RMN $-{ }^{13} \mathrm{C}\left(\mathrm{DMSO}-\mathrm{d}_{6}, 100 \mathrm{MHz}\right)$ do éster metílico do ácido verongidóico (7)

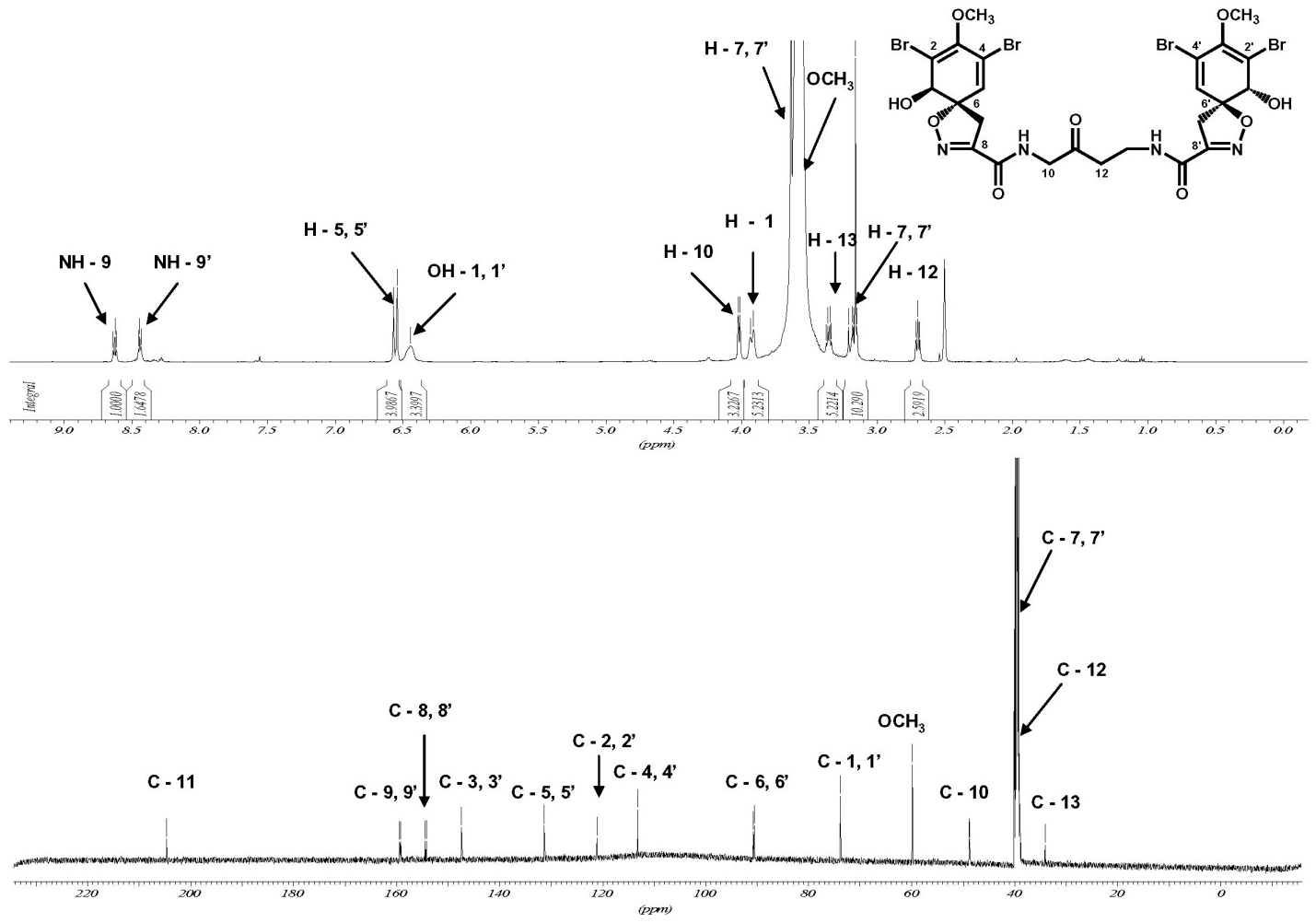

Figura 5S. Espectros de RMN $-{ }^{1} \mathrm{H}\left(\right.$ DMSO-d $\left._{6}, 400 \mathrm{MHz}\right)$ e RMN $-{ }^{13} \mathrm{C}\left(\right.$ DMSO-d $\left._{6}, 100 \mathrm{MHz}\right)$ da 11-oxoaerotionina (8) 


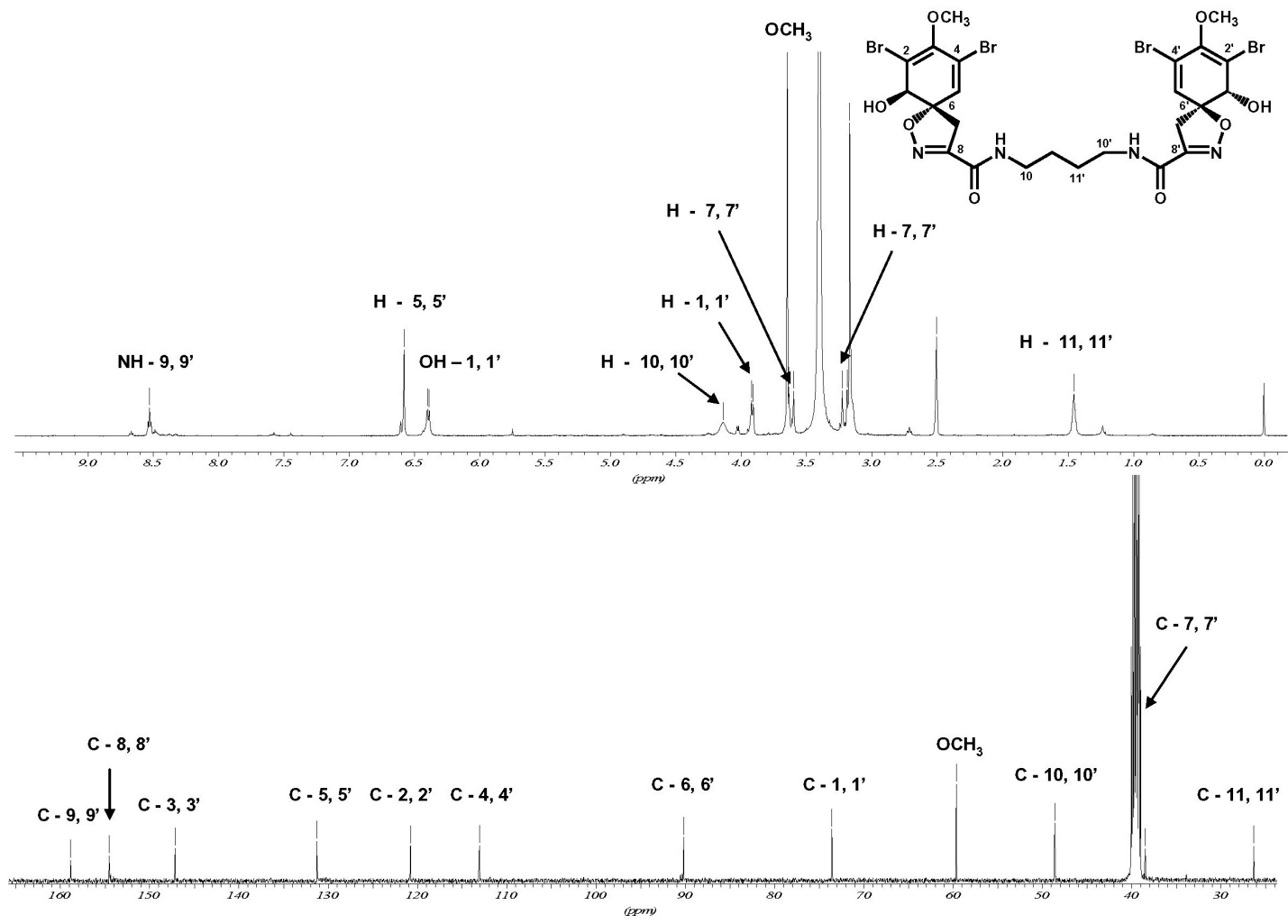

Figura 6S. Espectros de $R M N-{ }^{1} \mathrm{H}\left(\mathrm{DMSO}-d_{6}, 400 \mathrm{MHz}\right)$ e RMN $-{ }^{13} \mathrm{C}\left(\mathrm{DMSO}-d_{6}, 100 \mathrm{MHz}\right)$ da aerotionina (9)

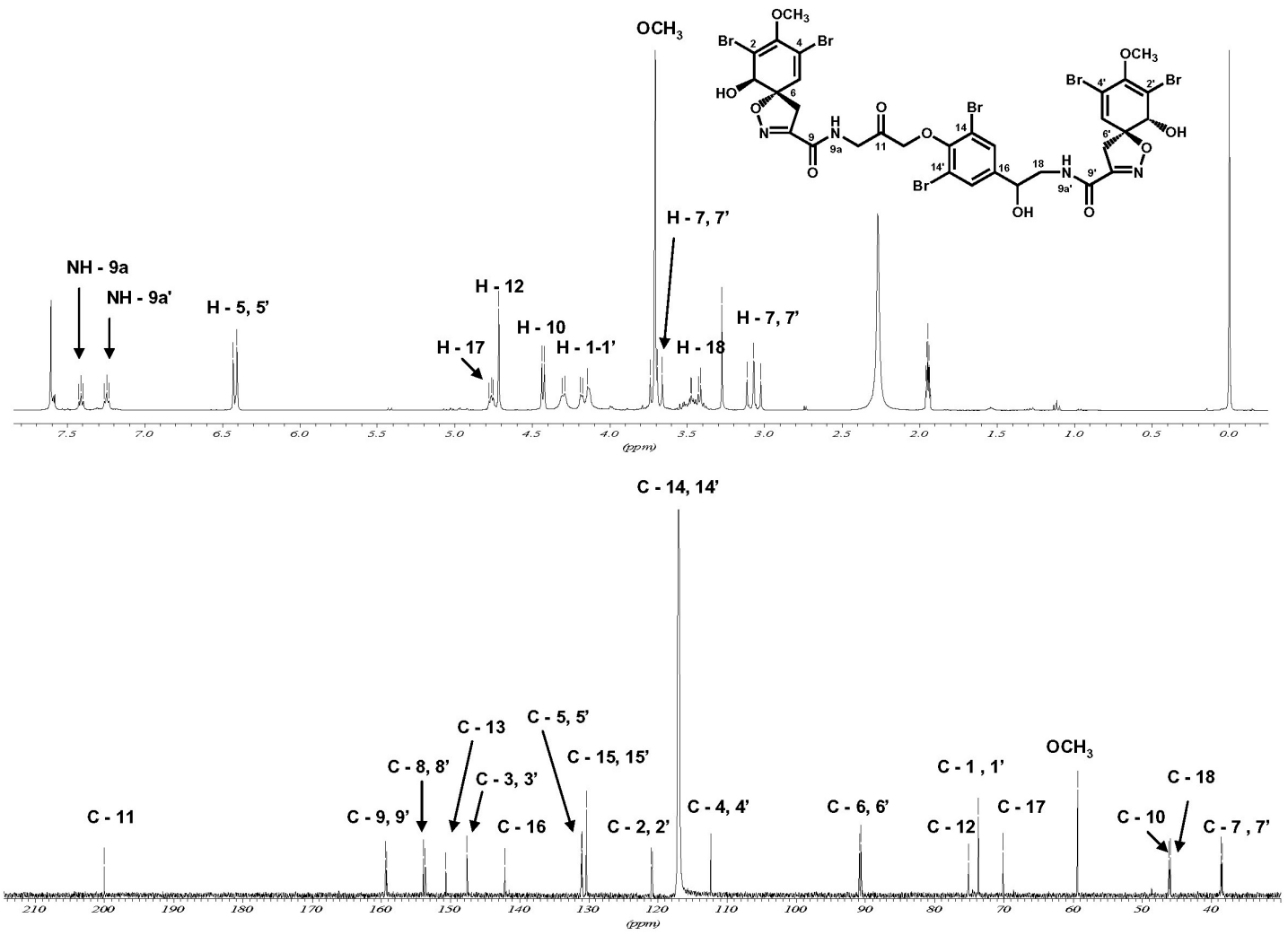

Figura 7S. Espectros de $\mathrm{RMN}-{ }^{1} \mathrm{H}\left(\mathrm{MeCN}-d_{3}, 400 \mathrm{MHz}\right)$ e $\mathrm{RMN}-{ }^{13} \mathrm{C}\left(\mathrm{MeCN}-\mathrm{d}_{3}, 100 \mathrm{MHz}\right)$ da 11-ceto-fistularina-3 (11) 

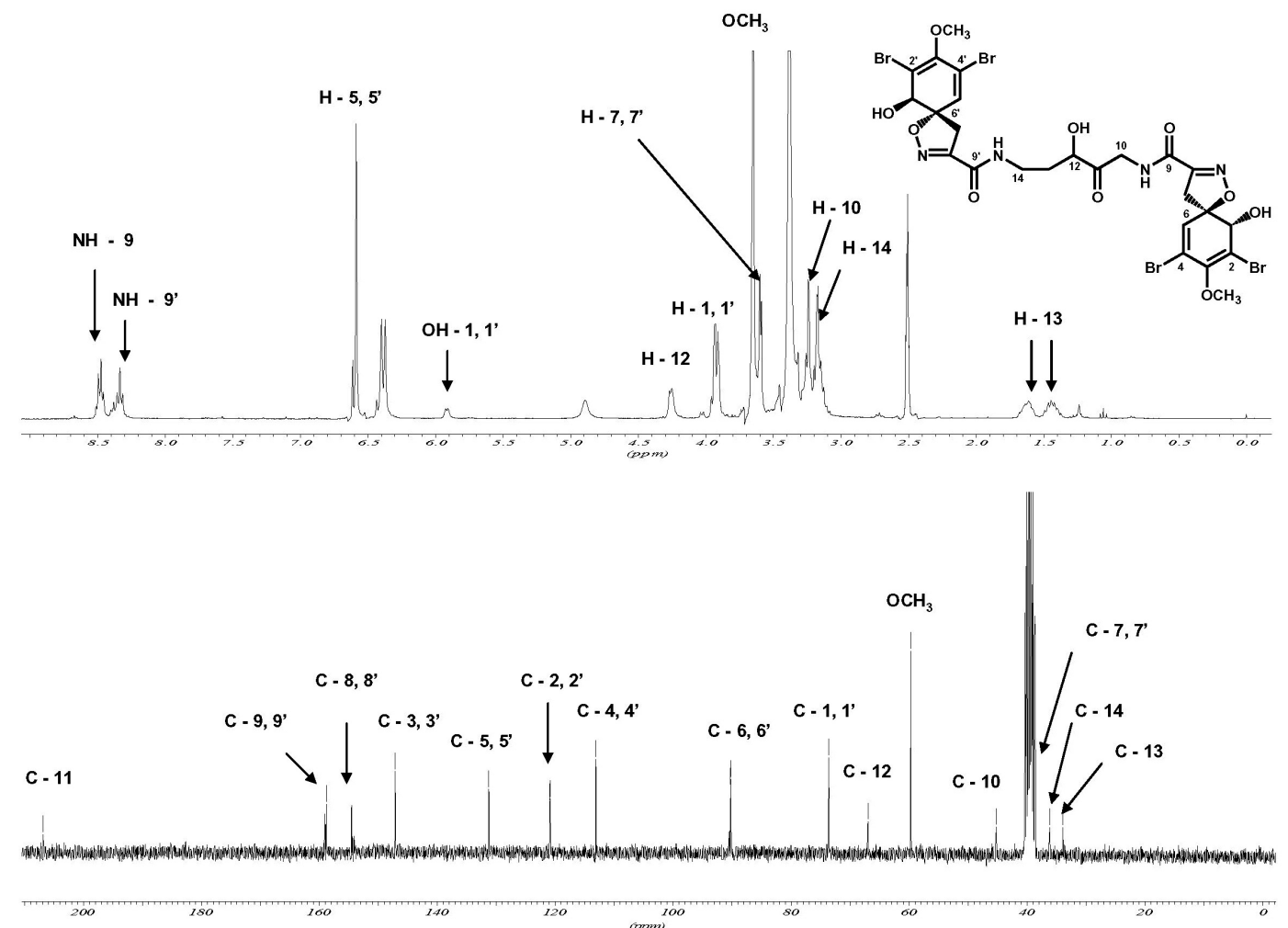

Figura 8S. Espectros de RMN - ${ }^{1} \mathrm{H}\left(\mathrm{DMSO}-\mathrm{d}_{6}, 400 \mathrm{MHz}\right)$ e RMN $-{ }^{13} \mathrm{C}\left(\mathrm{DMSO}-\mathrm{d}_{6}, 100 \mathrm{MHz}\right)$ da 11-oxo-12-hidroxi-homoaerotionina (10)

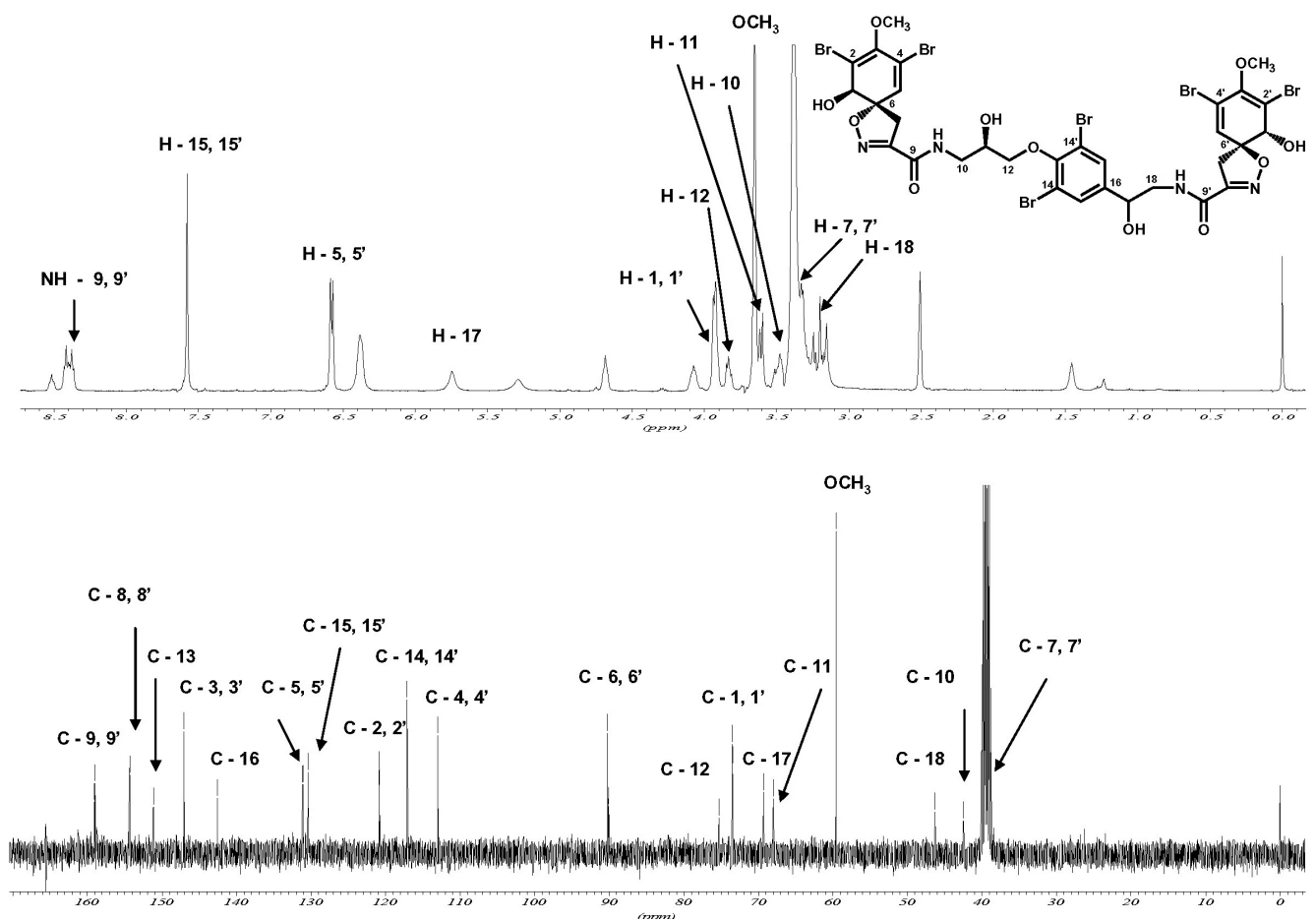

Figura 9S. Espectros de $R M N-{ }^{1} \mathrm{H}\left(\mathrm{DMSO}-\mathrm{d}_{6}, 400 \mathrm{MHz}\right)$ e RMN $-{ }^{13} \mathrm{C}\left(\mathrm{DMSO}-\mathrm{d}_{6}, 100 \mathrm{MHz}\right)$ da fistularina-3 (12) 